\title{
Incorporating Information from Trusted Sources to Enhance Urban Navigation for Blind Travelers
}

\author{
Byung-Cheol Min ${ }^{1}$, Suryansh Saxena ${ }^{2}$, Aaron Steinfeld ${ }^{1}$, and M. Bernardine Dias ${ }^{1}$
}

\begin{abstract}
Dynamic changes can present significant challenges for visually impaired travelers to safely and independently navigate urban environments. To address these challenges, we are developing the NavPal suite of technology tools [1]. NavPal includes a dynamic guidance tool [2] in the form of a smartphone app that can provide real-time instructions based on available map information to guide navigation in indoor environments. In this paper we enhance our past work by introducing a framework for blind travelers to add map/navigation information to the tool, and to invite trusted sources to do the same. The user input is realized through audio breadcrumb annotations that could be useful for future trips. The trusted sources mechanism provides invited trusted individuals or organizations an interface to contribute real-time information about the surrounding environment. We demonstrate the feasibility of our solution through a prototype Android smartphone-based outdoor navigation aid for blind travelers. An initial usability study with visually impaired adults informed the design and implementation of this prototype.
\end{abstract}

\section{INTRODUCTION}

The World Health Organization (WHO) estimates that 285 million people in the world are visually impaired, of whom 39 million are blind [3]. Although safe and independent mobility is a critical element of modern life, our needs assessment over the past few years [1] revealed that due to limited tools and information, traveling in unfamiliar environments can be challenging and often daunting for visually impaired people. We envision smart cities of the future that are truly accessible and navigable by everyone, including people with disabilities. These future smart cities are likely to benefit from assistive robots and a variety of other technology that could potentially enhance the safety and independence of visually impaired people during urban travel. However, smartphones are still likely to be the primary modality that connects visually impaired travelers to these other technology solutions and smart infrastructure in future smart cities.

To realize this vision of accessible smart cities, we are developing the "NavPal" suite of technology tools that combine a variety of technologies including robots, crowdsourcing, advanced path-planning and multi-modal interfaces to enhance the safety and independence of visually impaired people navigating urban environments [1], [2]. A key component of our work on accessibility and navigation is our commitment to a human-machine solution where humans and technology work together to achieve the best outcomes possible in any given

\footnotetext{
${ }^{1}$ Byung-Cheol Min, Aaron Steinfeld, and M. Bernardine Dias are with the Robotics Institute at Carnegie Mellon University, Pittsburgh, PA 15213 USA bmin@cs.cmu.edu, steinfeldecmu.edu, mbdiaseri.cmu.edu

${ }^{2}$ Suryansh Saxena was affiliated with Carnegie Mellon University when this work was done and is now with Delhi Technological University, New Delhi, Delhi, 110042 India suryanshered-itech. com
}

scenario. In this paper, we explore three ways in which blind users can be empowered to influence their assistive navigation aids - first, by adding audio annotations for themselves to use at future times; second, by choosing to share useful information with others who invite them as trusted sources; and third, by inviting trusted sources of relevant information to influence their navigation decisions. The contribution of this work is therefore a framework to empower blind travelers to influence their technology-based navigation aids via audio breadcrumb annotations and relevant information from userdesignated trusted sources.

The remaining sections of this paper are organized as follows. In section II, related work from the literature is discussed. Our system architecture and iterative design process are presented next in sections III and IV respectively. In section $\mathrm{V}$, we describe our mechanism to insert audio breadcrumbs and to invite trusted sources. Sections VI and VII detail relevant use-case scenarios for audio breadcrumb annotations and trusted sources. Finally, section VIII concludes this paper with a summary of the presented work, a discussion of the strengths and limitations of our approach, and an outline of relevant future work.

\section{RELATED WORK}

Assistive technologies have played an essential role in the safety and independence of people with disabilities. Long white canes, dog guides [4], and smartphones [2], [8], [9] are current assistive tools that are widely used by visually impaired people to aid in navigation. Several research efforts have also created a variety of guide robots [5], [6], [7] for this purpose.

A white cane is the most ubiquitous and universally identifiable navigation tool for visually impaired travelers and is used to detect and avoid obstacles and warn others in the path of travel [10]. A dog guide is another modality that visually impaired travelers often employ for the purpose of guidance in navigation. A white cane and dog guide have proven useful, but do not include a point-to-point navigation (route planning from a starting point to the intended destination) capability that is often necessary for visually impaired people to navigate safely and independently in unfamiliar environments.

In recent years, the use of smartphones has grown rapidly in visually impaired communities as they become more affordable and integrate several key functions needed for their daily life such as text-to-speech, gesture recognition, and localization using sensors. This follows similar trends showing rapid increases in smartphone market share [11]. 
Through the NavPal project [1], [2], [9], [13], we have been working on smartphone-based localization and navigation techniques to empower blind travelers with enhanced safety and independence when navigating indoors. Similarly, representative smartphone GPS apps targeted toward visually impaired users include BlindSquare [12] and Ariadne GPS [14]. BlindSquare is a very popular app running on the iOS platform. It uses points of interest information from Foursquare and Open Street Map and allows navigation to the point of the interest. Loadstone GPS is GPS navigation software for visually impaired users [15]. This software runs on Nokia mobile phones and uses external devices such as screen readers and a Bluetooth GPS receivers. The primary challenge for such commercial navigation solutions stems from their use of proprietary datasets to provide map information. Because of this, visually impaired users often need to pay to run those apps on their smartphones and require continuous updates on a map that often incurs additional charges. Moreover, most route planning apps are inflexible to adapt to dynamic changes, for example, blocked travel due to construction, and are not designed to be updated and refined by users.

In recent years, a growing number of research groups have shown interest in the use of crowdsourcing techniques to provide information about unfamiliar environments to enable blind travelers to navigate safely [16], [17]. Coughlan and Shen proposed a crowdsourcing approach to allow volunteers to enter information for providing guidance to blind travelers at traffic intersections [16]. The motivation of the approach is that important information such as the presence and location of walk buttons may be unavailable from some existing data sources such as Google Map, Open Street Map and other municipal/transit sources. VizWiz [17] is a smartphone app that uses a combination of image processing techniques and crowdsourcing using web workers and online social networks to allows visually impaired users to get nearly real-time answers to visual questions on their surroundings. However, as information for navigation aids is mainly from crowdsources in these approaches, it is hard for a user to have the ability to discern if the information is trustworthy and also it limits the user's options to get relevant information.

More relevant to our work is the Blind Leading the Blind project [18] that proposed the formation of online social networks where visually impaired travelers and sighted travelers collaboratively share and manage verbal route information, thus enabling visually impaired travelers to independently travel to previously unknown environments when provided with online verbal route directions. Similarly, Volkel et al. proposed a concept of sharing relevant Points of Interest (POI), such as temporary obstacles, for mobility impaired pedestrians [19]. In a similar context, Karimi et al. proposed a social navigation network, called SoNavNet, where users are connected to each other to share navigation information that is based on user experiences [20]. Although all these approaches are applicable to and are designed for empowering visually impaired persons with navigating unfamiliar environments safely and independently, they do not allow visually

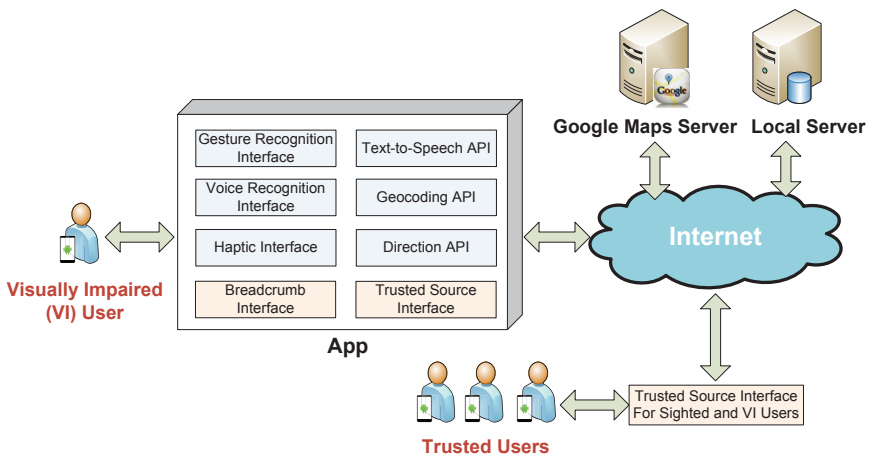

Fig. 1: System architecture of our app.

impaired users to invite or designate trusted individuals to and in social networks. Our previous needs assessments with visually impaired communities and orientation and mobility (O\&M) experts revealed that such technologies would help maximizing advantages of using such social networks for visually impaired travelers [1].

The relevant findings from our previous needs assessments are summarized as follows. First, visually impaired users prefer to invite trusted individuals and organizations to contribute information to assist their navigation. Next, visually impaired travelers wish to verbally annotate their routes on maps so they can record other information that will be useful for future trips to the same location. While different visual impairments and navigation techniques sometimes make it difficult to share entire paths or landmarks among visually impaired people in meaningful ways, most visually impaired travelers are happy to share navigation information with others who may find it useful. We address these issues in this paper via a mechanism for inviting trusted sources and inserting audio breadcrumbs.

\section{System ARCHITECTURE}

We demonstrate the feasibility of the proposed mechanism through a prototype Android smartphone app to assist blind travelers when navigating in outdoor urban environments. The system architecture of this app is illustrated in Fig. 1. The proposed system is composed mainly of two user groups (a visually impaired user and trusted users), Android smartphone app and interfaces for the groups, servers to store data, and Internet. Through Internet data from users and trusted sources are shared and map/route/direction information are kept upto-date.

User input to our app can be communicated in two modalities: (1) simple gestures on the touchscreen, or (2) voice input via the microphone. This multimodal input ensures that users are able to communicate with the app effectively even in a place where certain input modules do not work optimally (e.g., voice input in crowded places). The system's output to the user is communicated via synthesized speech and vibration feedback. Synthesized speech is generated by a text-to-speech (TTS) API that is available through the Google Library, and the TTS API converts text and messages displayed on the screen into voice output. A headset can allow a user to hear 


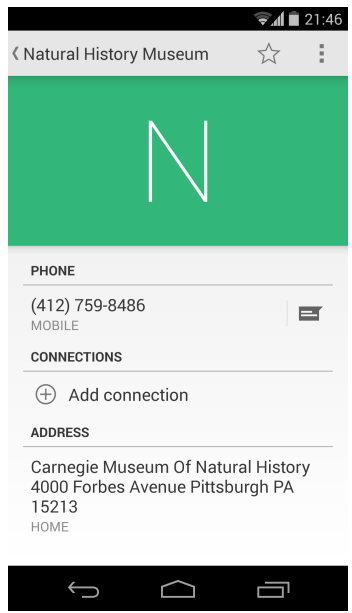

(a) Contact name and address

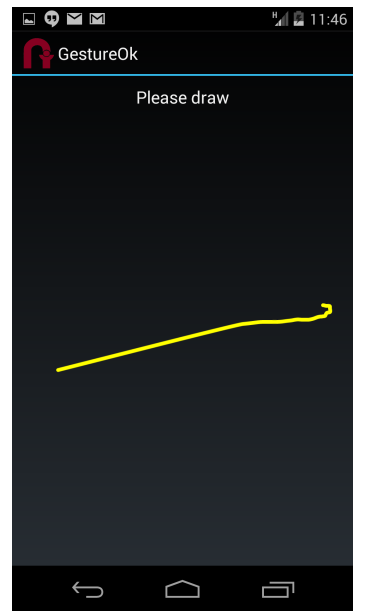

(b) Gesture drawn on the screen
Fig. 2: Snapshots of the contact destination and gesture input.

voice output in noisy environments. Vibration feedback via the haptic interface is used to acknowledge user input and also to warn users about potentially problematic situations, e.g., when the user diverges from the desired path during navigation.

Our app allows the user to select a contact name and address of the place or person as the intended destination. This feature is designed to allow visually impaired users to maintain their routine when placing a call to others using their smartphones. When a destination is received from the user in the form of the contact, e.g., "Natural History Museum", a full address is then retrieved, e.g., "Carnegie Museum of Natural History 4000 Forbes Avenue Pittsburgh PA 15213", which is needed for the Geocoding API that converts this address to geographic coordinates (latitude 40.443276 and longitude -79.950082) via an HTTP request to the Google Maps server. Fig. 2 (a) shows a snapshot of this example with the contact name displayed on the top and the contact address displayed on the bottom. The Google Maps server returns an output in JSON (JavaScript Object Notation). While a route could be directly planned with text strings of the address, geographic coordinates can represent locations with a much finer resolution so we used geographic coordinates for route planning in our app. This higher resolution is greatly beneficial to blind travelers, especially when they are navigating as pedestrians. Next, a route is planned from the current position to the intended destination using a Direction API that returns multi-part directions using a series of waypoints in JSON from the Google Maps server.

Fig. 3 shows an illustration of interactions between our app and the user. The text strings displayed in the boxes on the right represent the steps in the user's interaction. The text strings displayed in the boxes on the left represent the audio messages the app returns to the user.

Starting from the top right, the user launches the app on the smartphone and receives a verbal request to select a transportation mode from choices such as walking, public

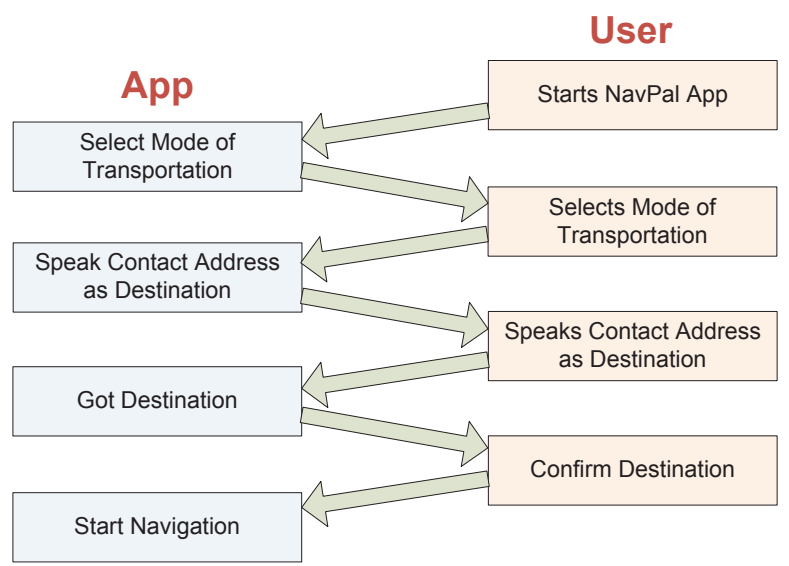

Fig. 3: Interactions between our app and the user.

transport, and car. Upon receiving the selected transportation mode, either from the gesture interface or speech interface, the app verbally announces that an input has been received. Next, the user is verbally asked to input the contact representing the desired destination. It should be noted that the address field should be filled in along with the contact name within the contact list of the smartphone device beforehand for the app to work properly. If a contact does not have an address stored beforehand, then the app will return an address as Null and ask the user to try again. The user then receives an audible message indicating the app is ready for navigation. If the user is in a location where the GPS signal is weak or unavailable, the app asks the user to change locations until the GPS signal is strong. Once the user is ready to navigate, he/she executes the relevant on-screen gesture to confirm this state and initiate navigation. While navigating with the app, the user is given audible navigational instructions at different waypoint intervals, e.g., "Head north for 20 meters and then turn left". In addition, the street name and user's direction of travel are announced at intersections. The nearby points of interest are also automatically announced to the user for better localization and orientation. If the user deviates from the desired path at a setting defined by us, e.g., 10 meters, the app directs the user to stop and plans a new path to the destination.

\section{Iterative Design with Visually IMPaired Users}

\section{A. Input via on-screen gestures}

On-screen gestures are commonly used input modalities for users who are blind or visually impaired to use a touchscreen smartphone, and thus we adopted gestures as part of our accessible interface. The literature [21], [22] shows that some gestures are easier than others for users who are blind or visually impaired. In this paper, we conducted a short usability study to determine which gestures are more effective for our app with relatively older participants $(n=4)$ than the participants in the literature. While the average age of our participants is 63.5 , the average age of blind participants $(n=10)$ in [21] is 49.0 , and ages of sighted but blindfolded 


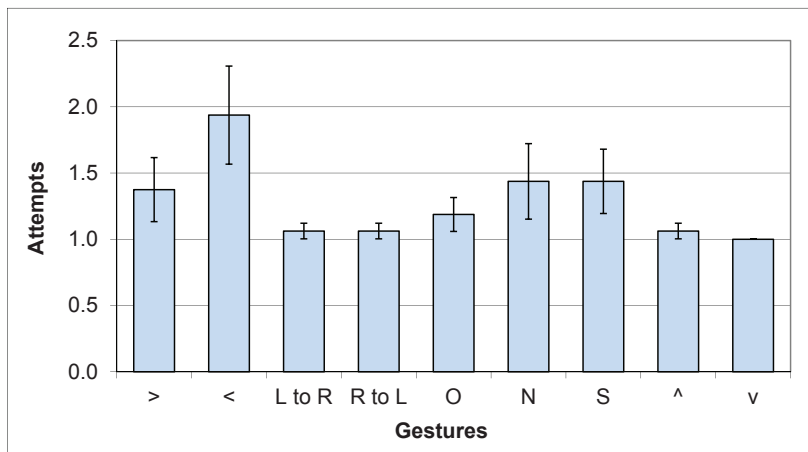

Fig. 4: Summarized results from gesture testing. Note that the lower attempts indicate that participants performed the gesutre more correctly.

participants $(n=12)$ in [22] range between 22 and 34. Our participants' ages ranged from 61 to $66, \mathrm{P} 1, \mathrm{P} 2$, and $\mathrm{P} 3$ are male and own iPhones or iPads, and $\mathrm{P} 4$ is female and owns no touchscreen devices. P1, P3, and P4 are blind and P2 is legally blind. For this usability study, we employed a Samsung Nexus $S$ that has a 4.0 -inch screen. It would be worth noting that this study would have the limitation of not gathering data on if the participants were right-handed or left-handed since that could affect performing certain gestures.

In our previous studies in [13], we conducted user studies with 8 visually impaired participants on various gestures on a touchscreen smartphone. Although we could not derive which gestures are easier or more difficult in a numerical fashion, the studies have shed on selecting the following eight gestures for the gesture testing for our app: $>,<$, swipe right, swipe left, $\mathrm{O}$, $\mathrm{N}, \mathrm{S}, \wedge$, and $\vee$. First, we walked the participant through each gesture teaching them how to perform them. Then, we directed them to attempt specific gestures which the app communicated to them one at a time. Participants could have up to five attempts on each gesture so that we could discern a quantitative score for difficulty of each gesture without overly taxing the users in terms of time or frustration. Once participants performed a specified gesture correctly, they were advanced to the next gesture. Once participants completed the list of nine specified gestures, they were asked to attempt the list again in a different order. The order of gestures was randomly selected by the app to minimize any bias in performance due to gesture ordering. The test concluded once this list was completed five times (with the list of gestures presented in five different configurations) by each participant. Fig. 4 shows the average with the standard deviation of attempts for each gesture over participants. Note that the lower attempts on a vertical axis indicate that participants performed the gesture more correctly. From this figure, we can see that the most troublesome gesture was $<$, followed by $\mathrm{N}$, then $\mathrm{S}$, then $>$. Also, we can see that $\mathrm{N}$ and $S$ which have motions in multiple directions were incorrect more often. These findings are consistent with the participant comments which reflected a greater difficulty and effort (in terms of time spent) to execute these gestures.

Fig. 4 further shows that the gestures swipe right, swipe left, $\wedge, \vee$, and $\mathrm{O}$ were the best executed gestures. However, $\wedge$ and $\vee$ were not always drawn accurately. More often than not they were executed more as L shapes following the sides of the screen. This result indicates that $\mathrm{L}$ shapes might be a good gesture to use since visually impaired users can follow the screen edge to write that gestures although it may require a greater effort in terms of time spent. All participants recommended using simpler gestures instead of ones that have components that go in multiple directions. Commonly recommended gestures were directional swiping with one or two fingers, which is consistent with what we discovered in past work [13], and therefore we selected those directional swiping gestures for our app as shown in Fig. 2 (b).

\section{B. Overall accessibility of interface}

Accessibility and ease of operating the app and adding data to it (via voice and gestures) are critical for visually impaired users. Therefore, we also conducted usability studies to assess the accessibility of the interface with the same four visually impaired participants. To test the accessibility of our app, the participants were first given a demo of the app interface, and were then asked to use either gestures or speech to input the transportation medium that they would use to navigate, and the contact name of the place/person to which they plan to navigate (e.g., Natural History Museum). Participants were also asked to use gestures to give system answers and feedback as requested by the app. This testing process (Test 1) was repeated twice for each participant. Furthermore, to test ease of use of the interface, participants were asked to speak an address location to annotate, and to use gestures to navigate the map and add a waypoint (Test 2). The results from these tests are summarized in Table I that shows how many were successful from the $\mathrm{n}$ attempts.

TABLE I: USABILITY STUDIES ON OUR APP

\begin{tabular}{|c|c|c|c|}
\hline \multirow{2}{*}{ Participant } & \multicolumn{2}{|c|}{ Test 1 } & Test 2 \\
\cline { 2 - 3 } & $(\mathbf{n = 3})$ & $\mathbf{( 3 )}$ & (2) \\
\hline P1 & 3 & 3 & 2 \\
\hline P2 & 1 & 2 & 2 \\
\hline P3 & 2 & 3 & 2 \\
\hline P4 & 0 & 2 & - \\
\hline Average & 1.5 & 2.5 & 2 \\
\hline
\end{tabular}

P4 was new to using a smartphone device, and thus had difficulties figuring out the gesture interaction with the device in the first attempt. But, P4 showed improvement in the second attempt. This participant was also unable to complete the latter part of the experiment due to scheduling constraints which required $\mathrm{P} 4$ to end the test session early.

Following these tests, we also conducted a debrief session where we collected feedback from the participants (verbally) about their experience during the quick tests, and their feedback on [a] speech recognition, [b] voice instruction, [c] gesture instruction, and [d] overall ease of use and accessibility of our app. We asked them to rate [a] to [c] in a range of 0 to 5 , with 0 being very bad and 5 being excellent, and [d] in a range of $0-10$. There was no specific reason to have these different rating ranges; but, we wanted to open up users' rating 
on overall performance of the app more widely. The results are summarized in Table II.

TABLE II: USER SCORE ON OUR APP

\begin{tabular}{|c|c|c|c|c|}
\hline $\begin{array}{c}\text { Participant } \\
\text { /Item }\end{array}$ & $\begin{array}{c}{[\mathbf{a}]} \\
\text { Speech }\end{array}$ & $\begin{array}{c}{[\mathbf{b}]} \\
\text { Voice }\end{array}$ & $\begin{array}{c}{[\mathbf{c}]} \\
\text { Gesture }\end{array}$ & $\begin{array}{c}{[\mathbf{d}]} \\
\text { Overall }\end{array}$ \\
\hline P1 & 5 & 4 & 5 & 9 \\
\hline P2 & 5 & 3 & 5 & 8 \\
\hline P3 & 4 & 4 & 5 & 9 \\
\hline P4 & 2 & 4 & 4 & 6 \\
\hline Average & 4 & 3.75 & 4.5 & 8 \\
\hline
\end{tabular}

The system had difficulty understanding the speech inputs by $\mathrm{P} 4$ because he/she is more accustomed to typing on a physical keyboard with a talk-back feature. This caused P4 to assign a score of 2 out 5 on the speech function. However, on average, the participants liked the overall system and its features, but commonly pointed out that we would need to implement a verbosity tool for avoiding repetitive instructions. This verbosity comment is reflected in the relatively low scoring for the voice instruction feature. Also, all participants, when presented with our planned methodology for introducing audio breadcrumbs and an interface to incorporate information from trusted sources, wholeheartedly approved of the plan and indicated that it would be very helpful. Selected comments from this user feedback are shown below.

- "The breadcrumb approach will be helpful for the user for orienting and locating in frequently visited places." (P3)

- "Trusted sources will be very helpful not only for outdoor navigation but also for indoor navigation. For example, trusted sources can help visually impaired users to read the room number or the direction." (P2)

\section{Audio Breadcrumbs and Trusted Sources}

While most of the online map services including Google Maps provide information needed for route planning, the map rarely provides continuous information about dynamic changes, for example blocked street and sidewalk, which are often useful for blind travelers to accomplish safe and independent navigation. In addition, these solutions do not allow users to verbally annotate their routes on the maps. Addressing these shortcomings, we present a framework for incorporating information from trusted sources, and user annotations in the form of audio breadcrumbs to enhance assistive navigation aids.

The underlying motivation for a framework that incorporates input from trusted sources is enabling individuals and organizations trusted by visually impaired travelers to share their observations and information relevant to navigating unfamiliar environments via a connected network. This collaborative information sharing will ultimately empower blind travelers to navigate unfamiliar urban environments safely and independently. For example, if a street is under renovation and a trusted source traveling via this route observes this potential risk to blind travelers, he/she can report this information using the trusted sources interface. A blind traveler can then benefit from this information since the route planner can take this information into account and provide an alternative path to the destination.

Trusted sources can be relevant authorities in the locality (e.g., a policeman), property managers (e.g., a business owner), O\&M experts, family and friends, or visually impaired travelers themselves. Previous studies [23] have shown that many visually impaired people prefer obtaining navigational information from other visually impaired persons because they are more likely to get information and directions that are accessible and relevant to them. Therefore, we made sure that visually impaired people can also act as trusted sources to other blind travelers. However, since there are many sighted people who are either trained or have learned to give good directions to blind travelers, we also made sure that the trusted sources interface was accessible to sighted individuals.

Furthermore, a visually impaired traveler can verbally record points of interest and reminders during a navigational experience and refer to it for future trips using the audio breadcrumb interface. The user can launch with a long tap (longer than 2 seconds) on the screen to record a message. When using this interface, the user can record a message and play it back. Examples of the message would include any potential problems encountered at that location, a waypoint identifier, or orientation information for future trips. The audio breadcrumb then appears as a waypoint on the map and as the traveler approaches the vicinity of this waypoint, the recorded information is played back to the user.

Visually impaired users are given two options when using the trusted sources interface. The first option is to use the current location as the tag for the recorded information. The second option is to specify a location other than the current location and associate it with the recorded information. If they select to use the current location, then the breadcrumb interface is launched and the same procedure described above is followed. If the user instead selects to add some other

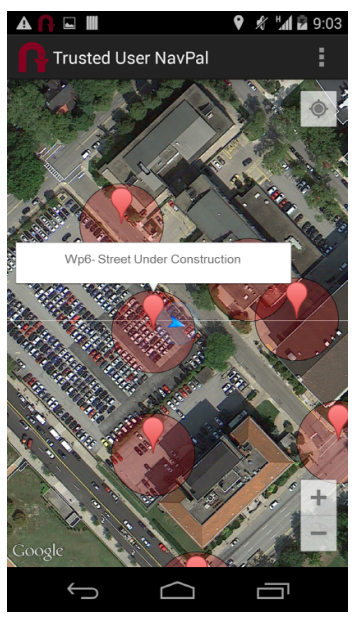

(a) Information added by a sighted trusted source

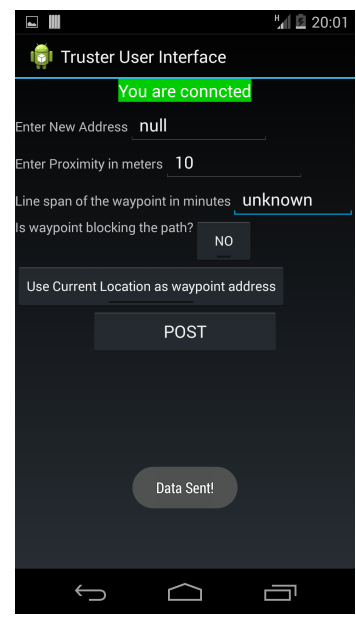

(b) Trusted source interface for sighted users
Fig. 5: A snapshot of the trusted source interface for sighted users. 
position, then the app asks the user to either speak the location address or physically type the address of the waypoint via the talk-back keyboard interface. The user is then provided with the same instructions as in the scenario where the current position is selected as the waypoint tag.

For the sighted trusted users, we developed an additional app where they can simply tap the map on the screen and annotate any observed dynamic changes. Fig. 5 (a) shows a snapshot of the contributed data with an annotation "Street under construction" using the trusted source interface for sighted users shown in Fig. 5 (b). Fig. 5 (b) also shows that trusted users can specify attributes for the data such as whether or not the waypoint is an obstacle that blocks the path, the waypoint proximity (the "zone" that qualifies as having arrived at the waypoint), for example 5-10 meters, 10-15 meters, 15-20 meters, and an estimated lifetime for this entry. The estimated lifetime for a data point can be a fixed duration, or can be tagged as unknown.

Regardless of whether the trusted source is visually impaired or sighted, the information they provide is then directly sent and stored on a local server and is retrieved by our app if that source is selected as a trusted source by a user of the app. Using the trusted source interface, the blind traveler can select and designate specific contacts as trusted sourced, and thereby subscribe to all their updates. This list is stored in their Android internal memory and can be edited by selecting the setting option of this interface.

\section{USE-CASE SCENARIO FOR AUdio BREADCRUMBS}

In this section we demonstrate the performance of the audio breadcrumb feature of our app by narrating the performance of the app in a relevant use-case scenario. Note however that the demonstrations were carried out by sighted users at this stage. The demonstration was conducted by physically navigating with the help of our app from the Newell Simon Hall building in the Robotics Institute at Carnegie Mellon University (in Pittsburgh, PA, USA) to the Carnegie Natural History Museum which is located a few blocks away. During this traverse we tested the performance of our app focused on the feature of adding audio breadcrumbs and verifying the app performance when the user deviates from the planned route.

The demonstration began by launching the app immediately outside Newell-Simon Hall. We selected the walking as the modality of travel, and provided verbal input "Natural History Museum" to specify the desired destination. Upon confirmation of our input, the app planned the best route for this traverse, plotted this route on the map, and incorporated relevant navigation information from all relevant sources as shown in Fig. 6. The system then started the navigation guidance process and provided the estimated time and distance to the destination, followed by the first navigation instruction. As we the navigation instructions, the app demonstrated the desired behavior by waiting until we approached the next waypoint before providing the next navigation instruction.

In order to test the breadcrumb interface, we stopped along the path near a bench shaded by a tree. We then recorded

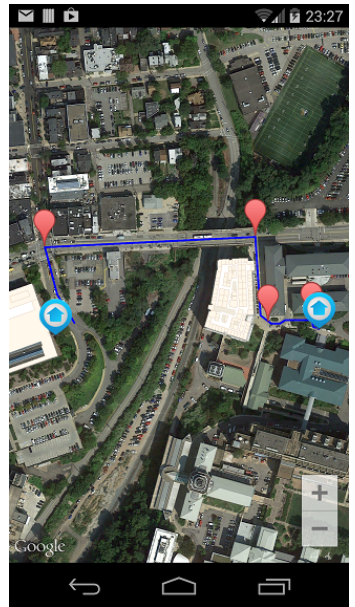

Fig. 6: Path planned connecting the origin to the destination using our app.

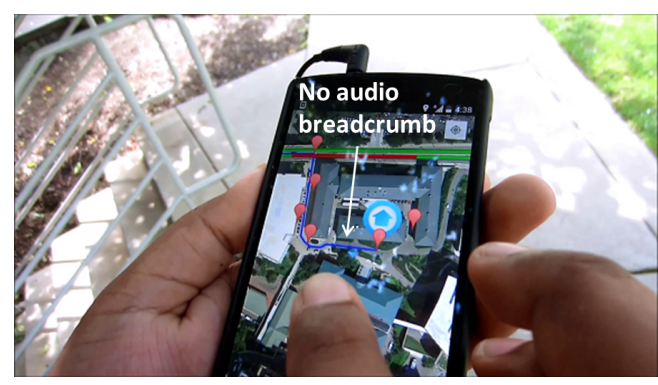

(a) Original map

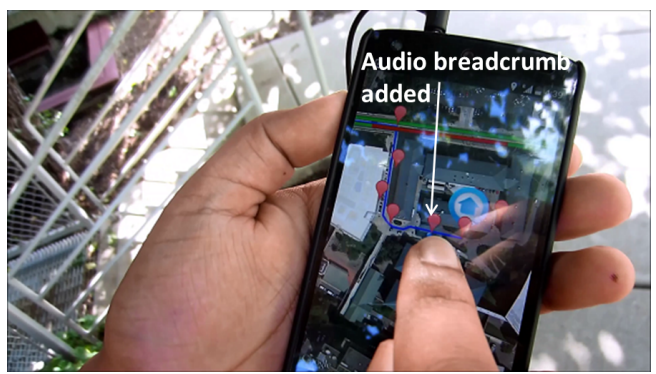

(b) Updated map

Fig. 7: Audio breadcrumb annotation - (a) The smartphone screen displaying the original map with five waypoints on the planed path; (b) The updated map with the breadcrumb added as a waypoint on the planed path

an audio breadcrumb annotation that indicated this was a good shady spot to take a seat and rest if we wished. After confirming the recorded message, the system added the audio breadcrumb as a new waypoint (shown in Fig. 7 (b)) along the originally planned route on the map (shown in Fig. 7 (a)).

To further test the performance of our app, we intentionally deviated from the recommended route near the destination waypoint. Specifically, when the system guided us to take a left turn, we intentionally took a right turn from that waypoint. The system immediately detected the deviation and provided a warning that we were moving in the wrong direction. When we continued in the incorrect direction the app asked us to stop and initiated a re-planning cycle to calculate a new route to our destination. The system re-routed after few seconds and 
correctly guided us to the destination.

Finally, we further verified the functionality of the audio breadcrumb annotation by using our app to guide us back to our original location, Newell Simon Hall. Upon confirmation of the desired navigation plan, the app started instructing us to navigate back to Newell-Simon Hall. We followed the instruction to reach the intended destination. As we approached the previously added breadcrumb, the recorded data was played back. This demonstrated that the breadcrumbs interface worked as expected. A video demonstrating our app can be found at https://www.youtube.com/watch?v=h8qs860Ezxo.

\section{USE-CASE SCENARIO FOR TRUSTED SOURCES}

In this section, similar to the previous section, we narrate the performance of our app in a relevant use-case scenario to demonstrate the performance of the app interface for trusted sources to add relevant navigation information. Similar to the previous demonstration scenario, this scenario was also executed by sighted users at this stage. In order to also show that our app performance is not location-dependent, the scenario for demonstrating the functionality of our app's interface for trusted sources was executed in Noida India. The demonstration began by physically navigating with the help of our app from the B Block Sector 26 Noida to the destination, "Kailash Healthcare Hospital" at H block sector 27. This route was chosen because a house near the destination on the route was under construction, and the sidewalk near this house was strewn with debris which could be hazardous for blind travelers. Fig. 8 shows the sites chosen for this demonstration.

When we were in the vicinity of the debris-strewn sidewalk, we launched the trusted user contribution interface and filled out attributes of the waypoint to inform blind travelers about this hazard. The first attribute was to indicate that the point of interest is not blocking the path of the user. Next, we added a waypoint proximity as 5-10 meters. Then, we recorded information for this waypoint similar to the breadcrumb approach. In this demonstration, we annotated this waypoint. The app then automatically established a connection with the local Google Application Engine (GAE) server and sent the new trusted data along with the cell phone number (CN1) which identifies the source. Next we used our app to launch a new navigation task with a second Android smartphone to demonstrate that the previously uploaded information can be correctly retrieved and played back when the navigator approaches the area. For this scenario, we pre-stored the initial phone number $(\mathrm{CN} 1)$ as a trusted source in the contact list of the second smartphone. To add the trusted source we used speech input to select the appropriate contact from our list and tag that contact as a trusted user. Once the contact was added to the trusted source list, the app established the link with the local GAE server and retrieved information submitted by that trusted source. This in turn triggered the addition of a new waypoint on the map, which incorporated the information provided by the trusted source as shown in Fig. 9 (b). With this updated map, we started navigation and the recorded

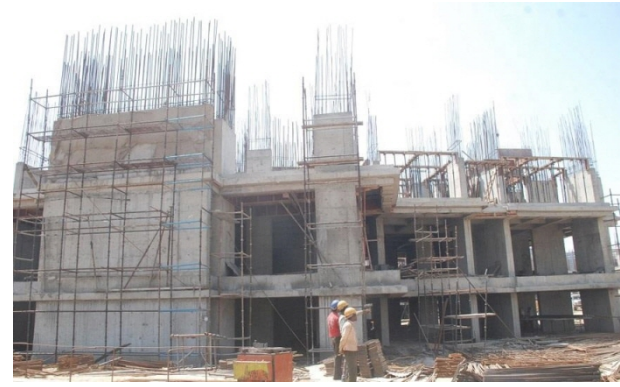

(a) House under construction

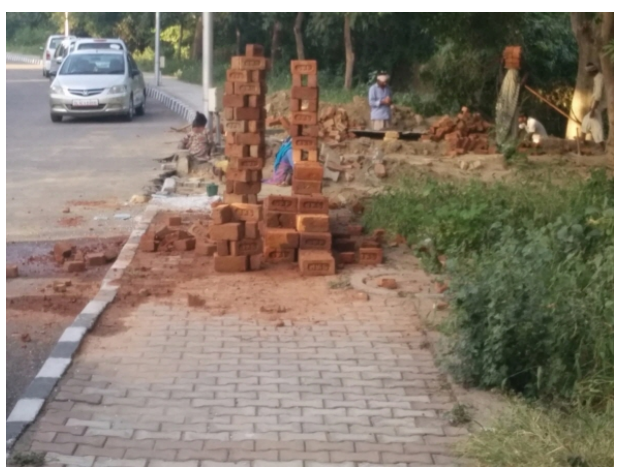

(b) Debris over the sidewalk

Fig. 8: Chosen locality for demonstrating our app's interface for trusted sources to provide navigation information included (a) a house under construction and (b) a sidewalk strewn with debris which made the path hazardous for blind travelers.

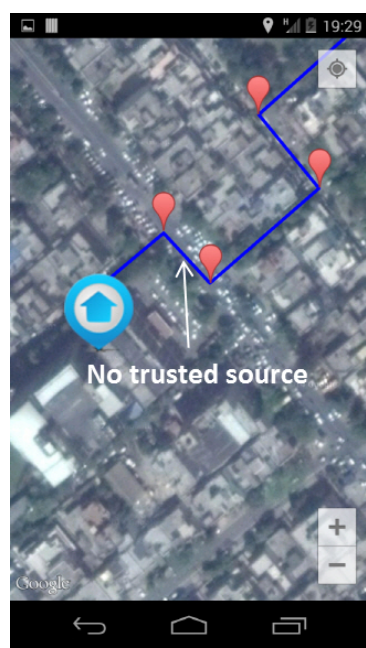

(a) Original map

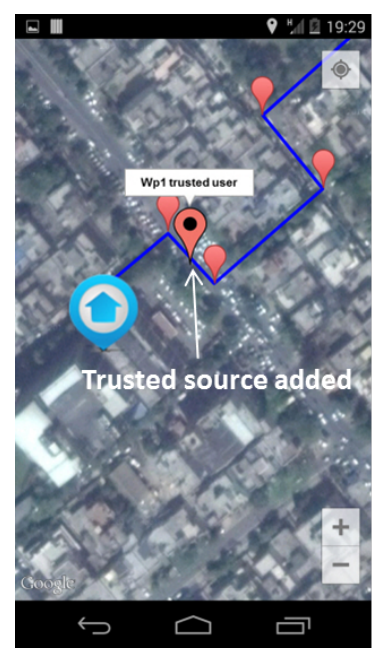

(b) Map updated with trusted source information
Fig. 9: Use-case scenario of trusted sources interface for our app - (a) shows the original map (b) shows the map updated with information from the trusted source shown as an additional waypoint

message was played back as we approached the corresponding waypoint.

\section{Discussion, CONClusions, AND FuturE WORK}

We strive towards a vision of smarter cities that are truly accessible to everyone including people with disabilities. To realize this vision, we are developing the NavPal suite of 
technology tools to empower blind and visually impaired travelers to safely and independently accomplish urban travel. In this paper we introduce two significant enhancements to the existing assistive navigation tools for this community. Namely, a mechanism for blind travelers to annotate their paths with useful information in the form of audio breadcrumbs, and a framework that allows user-designated trusted sources to provide navigation-related information that is incorporated into the route planning of the assistive tools. We developed a prototype smartphone-based navigation tool that demonstrates both of these enhancements, including a quick usability study to validate the concept and inform some aspects of the implementation. Moreover, we described detailed use-case scenarios that demonstrate the value of these enhancements.

While the demonstrated approach provides significant benefits to the target users, there are also a few limitations that must be addressed in future work. One consideration is the amount of data that the trusted sources can generate. Large amounts of data can slow down the responsiveness of the app and also could incur monetary costs depending on specific smartphone data plans. We are considering several data management strategies to address this concern. A second consideration is managing information conflicts from different trusted sources and managing information with unknown lifetimes. We can borrow strategies from the literature where a variety of technology solutions have grapples with similar issues. Other planned future work includes implementing verbosity control measures for avoiding repetitive instructions, enhancing localization accuracy, eliminating the dependence on the Google servers, and testing the app with visually impaired users.

\section{ACKNOWLEDGMENTS}

This work was sponsored by the National Science Foundation under NSF-NRI Award Number 1317989, and by the National Institute on Disability and Rehabilitation Research under the RERC-APT (grant number H133E080019). The authors also thank the participants in the user studies that informed this work, and the many members of the research team who assisted with different aspects of this work; notably Ermine Teves, Nick Hainsey, and Omar Mustardo. The Carnegie Mellon University Institutional Review Board has approved this study.

\section{REFERENCES}

[1] M. B. Dias, "NavPal: Technology Solutions for Enhancing Urban Navigation for Blind Travelers," tech. report CMU-RI-TR-21, Robotics Institute, Carnegie Mellon University, Sep. 2014.

[2] B. Kannan, N. Kothari, C. Gnegy, H. Gedaway, M. F. Dias, and M. B. Dias, "Localization, Route Planning, and Smartphone Interface for Indoor Navigation," in Cooperative Robots and Sensor Networks, A. Kouba and A. Khelil, Eds. Springer Berlin Heidelberg, 2014, pp. 39-59.

[3] "WHO - Visual impairment and blindness," WHO. [Online]. Available: http://www.who.int/mediacentre/factsheets/fs282/en/. [Accessed: 27-Feb-2015].

[4] "Guide Dogs of America." [Online]. Available: http://www.guidedogsofamerica.org/1/. [Accessed: 27-Feb-2015].
[5] I. Ulrich and J. Borenstein, "The GuideCane-applying mobile robot technologies to assist the visually impaired," IEEE Transactions on Systems, Man and Cybernetics, Part A: Systems and Humans, vol. 31, no. 2, pp. 131-136, Mar. 2001.

[6] G. Galatas, C. McMurrough, G. L. Mariottini, and F. Makedon, "eyeDog: An Assistive-guide Robot for the Visually Impaired," in Proceedings of the 4th International Conference on PErvasive Technologies Related to Assistive Environments, New York, NY, USA, pp. 58:1-58:8, 2011.

[7] V. Kulyukin, C. Gharpure, J. Nicholson, and G. Osborne, "Robotassisted wayfinding for the visually impaired in structured indoor environments," Auton Robot, vol. 21, no. 1, pp. 29-41, Aug. 2006.

[8] P. Narasimhan, R. Gandhi, and D. Rossi, "Smartphone-based Assistive Technologies for the Blind," in Proceedings of the 2009 International Conference on Compilers, Architecture, and Synthesis for Embedded Systems, New York, NY, USA, 2009, pp. 223-232.

[9] N. Kothari, B. Kannan, E. D. Glasgwow, and M. B. Dias, "Robust Indoor Localization on a Commercial Smart Phone," Procedia Computer Science, vol. 10, pp. 1114-1120, 2012.

[10] J. A. Hesch and S. I. Roumeliotis, "An Indoor Localization Aid for the Visually Impaired," in 2007 IEEE International Conference on Robotics and Automation, 2007, pp. 3545-3551.

[11] A. Smith, "Nearly half of American adults are smartphone owners," Pew Research Center's Internet \& American Life Project, 01-Mar-2012.

[12] B. Holton, "BlindSquare App for iOS: A Solution for Accessible Navigation," AFB AccessWorld Magazine, vol. 15, no. 7, Jul. 2014

[13] Hend K. Gedawy, "Designing an Interface and Path Translator for a Smart Phone-Based Indoor Navigation System for Visually Impaired Users." tech. report CMU-CS-11-125, Computer Science, Carnegie Mellon University in Qatar, Dec. 2011.

[14] "An innovative app for your monility." [Online]. Available: http://www.ariadnegps.eu/. [Accessed: 27-Feb-2015].

[15] "Loadstone GPS - Free GPS Software for our Mobile Phone." [Online]. Available: http://www.loadstone-gps.com/. [Accessed: 27-Feb-2015].

[16] J. M. Coughlan and H. Shen, "Crosswatch: a System for Providing Guidance to Visually Impaired Travelers at Traffic Intersections," $J$ Assist Technol, vol. 7, no. 2, Apr. 2013.

[17] J. P. Bigham, C. Jayant, H. Ji, G. Little, A. Miller, R. C. Miller, R. Miller, A. Tatarowicz, B. White, S. White, and T. Yeh, "VizWiz: Nearly Real-time Answers to Visual Questions," in Proceedings of the 23Nd Annual ACM Symposium on User Interface Software and Technology, New York, NY, USA, 2010, pp. 333-342.

[18] V. Kulyukin and J. Marston, "The Blind Leading the Blind: Toward Collaborative Online Route Information Management by Individuals with Visual Impairments," AAAI Spring Symposium: Social Information Processing, pp. 54-59, 2008.

[19] T. Völkel, R. Kühn, and G. Weber, "Mobility impaired pedestrians are not cars: Requirements for the annotation of geographical data", ICCHP '08 Proceedings of the 11th international conference on Computers Helping People with Special Needs, pp. 1085-1092, 2008.

[20] H. A. Karimi, B. Zimmerman, A. Ozcelik, and D. Roongpiboonsopit, "SoNavNet: a framework for social navigation networks." Proceedings of the 2009 International Workshop on Location Based Social Networks. ACM, pp. 81-87, 2009.

[21] S. K. Kane, J. O. Wobbrock, and R. E. Ladner. "Usable gestures for blind people: understanding preference and performance." Proceedings of the SIGCHI Conference on Human Factors in Computing Systems. ACM, pp. 413-422, 2011.

[22] D. McGookin, S. Brewster, and W. Jiang. "Investigating touchscreen accessibility for people with visual impairments." Proceedings of the 5th Nordic conference on Human-computer interaction: building bridges. ACM, pp. 298-307, 2008.

[23] J. Balata, J. Franc, Z. Mikovec, and P. Slavik, "Collaborative navigation of visually impaired," Journal of Multimodal User Interfaces, vol. 8, no. 2, pp. 175-185, Jun. 2014. 